\title{
Aplicação do método índice de susceptibilidade para estimativa da susceptibilidade à contaminação na Bacia hidrográfica dos rios Turvo, Santa Rosa e Santo Cristo Application of the IS method for estimation of susceptibility to contamination in the Rios Turvo, Santa Rosa and Santo Cristo River Basin \\ Willian Fernando de Borba' (1), Bruna Ruchel ${ }^{2}$ (D), Kananda Cabral Menegazzo ${ }^{1}$ (D), Leonardo Zuchetto Peil ${ }^{1}$ (D), Cristiano Niederauer da Rosa ${ }^{3}$ (c) \\ ${ }^{1}$ Universidade Federal de Santa Maria - UFSM, Linha 7 de Setembro, s/n, BR 386, km 40, CEP 98400-000, Frederico Westphalen (RS), BR (borbawf@gmail.com; kanandacabralmenegazzo@gmail.com; leo_peil@hotmail.com) ${ }^{2}$ Centro Universitário Internacional - UNINTER, Lucas do Rio Verde (MT), BR (brunaruchel2102@gmail.com) 3Universidade Federal do Rio Grande do Sul - UFRGS, Porto Alegre (RS), BR (cristianonrd@gmail.com)
}

Recebido em 7 de agosto de 2019; aceito em 6 de fevereiro de 2020

\section{Resumo}

Em razão do elevado nível de contaminação das águas superficiais e da consequente diminuição da reserva hídrica potável, os mananciais de águas subterrâneas se mostram uma alternativa para suprir a demanda de água para consumo humano. Em virtude disso, é essencial o monitoramento das fontes potenciais de contaminação que podem afetar a qualidade ou a quantidade de água disponível. Desse modo, este estudo visou identificar potenciais áreas e fontes poluidoras do Sistema Aquífero da Serra Geral na escala da Bacia hidrográfica dos rios Turvo, Santa Rosa e Santo Cristo. Para isso, foi empregada a metodologia do índice de susceptibilidade à contaminação a partir do cruzamento de uma série de variáveis (topografia, uso do solo, material do aquífero, distância até o lençol freático e recarga). Os resultados indicaram que a susceptibilidade variou de muito baixa $(4,89 \%$ da área) a elevada $(0,004 \%)$, com predomínio da classe moderada a baixa. Isso indica que a área apresenta condições favoráveis à contaminação em sua maior porção. Destaca-se que grande parte das áreas próximas às zonas urbanas apresentaram elevada susceptibilidade à contaminação. O presente estudo apresenta $\mathrm{o}$ alto potencial das ferramentas de geoprocessamento na determinação do índice de susceptibilidade e mapeamento de áreas passíveis de contaminação, capaz de aprimorar a gestão das águas subterrâneas e na elaboração de planos ambientais municipais.

Palavras-chave: Água subterrânea; Índice de susceptibilidade; Recarga; Sistema Aquífero Serra Geral.

\begin{abstract}
Due to the high level of contamination of surface water and the consequent decrease in drinking water reserves, groundwater sources are an alternative to supply the demand for water for human consumption. As a result, it is essential to monitor the potential sources of contamination that may affect the quality or quantity of water available. Thus, this study aims to identify potential polluting areas and sources of the Serra Geral Aquifer System on the hydrographic basin scale of the Turvo, Santa Rosa, Santo Cristo rivers. For this, the methodology of contamination susceptibility index was used, from crossing of a series of variables (Topography, soil use, aquifer material, distance to the water table and recharge). The results indicated that the susceptibility ranged from very low (4.89\% of the area) to high $(0.004 \%)$, with a predominance of moderate to low class. This indicates that the area presents favorable conditions for contamination in its greater portion. It is noteworthy that great part of the areas near the urban zones showed high susceptibility to contamination. This study presents the high potential of geoprocessing tools in determining the susceptibility index and mapping areas susceptible to contamination, able to improve groundwater management and the elaboration of municipal environmental plans.
\end{abstract}

Keywords: Groundwater; Susceptibility index; Recharge; Serra Geral Aquifer System. 


\section{INTRODUÇÃO}

Segundo Heath (1982), água subterrânea é a água na zona saturada que está sob pressão igual ou maior que a atmosférica. Nesse sentido, o tipo de material presente no subsolo desempenha um importante papel, seja no movimento da água subterrânea, seja na proteção do meio subterrâneo em relação às atividades antrópicas desenvolvidas na superfície, com ênfase na função filtro desempenhada pelo solo (Rebouças et al., 2002). Assim, as questões envolvendo a gestão da oferta $\times$ a demanda da água subterrânea vêm sendo alvo de inúmeros debates (Rebouças, 2008), principalmente em regiões com déficit hídrico.

Segundo informações da Agência Nacional de Águas (ANA, 2015b), as águas subterrâneas são utilizadas para o abastecimento de municípios, principalmente em áreas isoladas, ou, ainda, em grandes centros urbanos. Ainda conforme o Atlas de abastecimento de água (ANA, 2015a), 2.153 municípios brasileiros $(38,65 \%)$ são abastecidos por mananciais subterrâneos, e no Estado do Rio Grande do Sul, esse percentual representa $57,55 \%(n=286)$ dos municípios do estado. Com isso, destaca-se a importância dos mananciais subterrâneos no fornecimento de água, não somente no cenário estadual, mas também no cenário nacional, principalmente nas zonas rurais.

Guiguer e Kohnke (2002) citam diversas metodologias para avaliar a vulnerabilidade à contaminação de aquíferos, sendo o DRASTIC - D (Depht to water) R (Recharge) $\mathrm{A}$ (Aquifer media) S (Soil media) T (Topography) I (Impact of vadose zone) $\mathrm{C}$ (Conductivity hidraulic) - , proposto por Aller et al. (1987), uma das mais utilizadas. Porém, esse método é limitado em razão das dificuldades na determinação de alguns dos parâmetros necessários para a sua estimativa, por causa de extensivas visitas a campo, o que torna sua utilização economicamente inviável, se considerarmos os orçamentos de pequenos municípios ou, ainda, das equipes de pesquisa.

Ao verificar as dificuldades supracitadas na implantação do método DRASTIC, de Aller et al. (1987), Ribeiro (2005) simplificou o método original, considerando somente os parâmetros D, R, A e T e acrescentando o uso do solo (Land Use), ou simplesmente LU. Esse método indica o índice de susceptibilidade (IS) dos aquíferos à contaminação, principalmente em áreas agrícolas, sendo empregado por vários pesquisadores (Peralta et al., 2001; Peralta et al., 2005; Anane et al., 2013; Ribeiro et al., 2017). No Brasil, esse método foi utilizado com eficácia por Borba et al. (2016) e Lunardi (2018), sendo os principais trabalhos desenvolvidos até o momento nessa região.

Para Chrispim (2016), o IS foi desenvolvido com o objetivo de caracterizar a vulnerabilidade das águas subterrâneas à poluição agrícola, sendo este uma modificação do DRASTIC. O método simplifica o sistema hidrogeológico, em termos do número de variáveis importantes para a determinação da vulnerabilidade, e introduz um fator relacionado à ocupação do solo e à contaminação das águas subterrâneas (Francés et al., 2001). Em Portugal, esse método é amplamente utilizado em estudos que avaliam a contaminação da água subterrânea por nitratos.

A identificação das áreas suscetíveis à contaminação é de grande relevância no planejamento de atividades exploratórias que visam à redução do impacto ambiental, pois apresenta aos gestores de bacias hidrográficas ferramentas que complementam o licenciamento ambiental e protegem as águas subterrâneas. Por isso, este estudo visou determinar o IS no Sistema Aquífero da Serra Geral (SASG) na escala da Bacia hidrográfica dos rios Turvo, Santa Rosa e Santo Cristo, localizada em uma região com forte atividade suinícola, o que reflete em alto risco de contaminação dos mananciais (superficiais e subterrâneos).

\section{Caracterização da área de estudo}

A Bacia hidrográfica dos rios Turvo, Santa Rosa e Santo Cristo (U-30), conforme a Secretaria do Meio Ambiente do Estado do Rio Grande do Sul (SEMA, 2004), situa-se a norte-noroeste do Estado do Rio Grande do Sul, entre as coordenadas geográficas $27^{\circ} 07^{\prime}$ a $28^{\circ} 13^{\prime}$ de latitude sul e $53^{\circ} 24^{\prime}$ a $55^{\circ} 20^{\prime}$ de longitude oeste (Figura 1). Ela abrange a província geomorfológica Planalto Meridional, cuja área drenada corresponde a $11.056,23 \mathrm{~km}^{2}$ (SEMA, 2004). Freitas et al. (2012) e Nanni et al. (2005), em estudos prévios, identificaram essa região com alto potencial para produção de cargas contaminadoras, o que pode ser atribuído às expressivas atividades suinícolas (Governo do Estado do Rio Grande do Sul, 2011) e agrícolas (cultivos de soja, trigo e milho).

\section{Caracterização geológica e hidrogeológica}

Segundo Motter e Foleto (2010), a região do Uruguai está subdividida em dez unidades hidrográficas, entre elas a U-30, onde está a Formação Geológica Serra Geral — província magmática relacionada aos derrames de intrusivas que recobrem 1,2 $\times 10^{6} \mathrm{~km}^{2}$ da Bacia do Paraná, abrangendo toda a região centro-sul do Brasil e estendendo-se ao longo das fronteiras do Paraguai, do Uruguai e da Argentina (CPRM, 2004). Assim, essa bacia abrange uma área de rochas vulcânicas, onde a recarga desse aquífero ocorre, principalmente, pelas fraturas do basalto (Freitas et al., 2012).

Como é possível observar na Figura 2, a maior parte da bacia hidrográfica é constituída de rochas vulcânicas da Formação Serra Geral - Fácies Paranapanema (k1_beta_pr), composta de derrames basálticos granulares finos, melanocráticos, contendo horizontes vesiculares espessos preenchidos por quartzo, zeólitas, carbonatos, celadonita, cobre nativo e barita (CPRM, 2006). Tem-se também, em menor área, a 


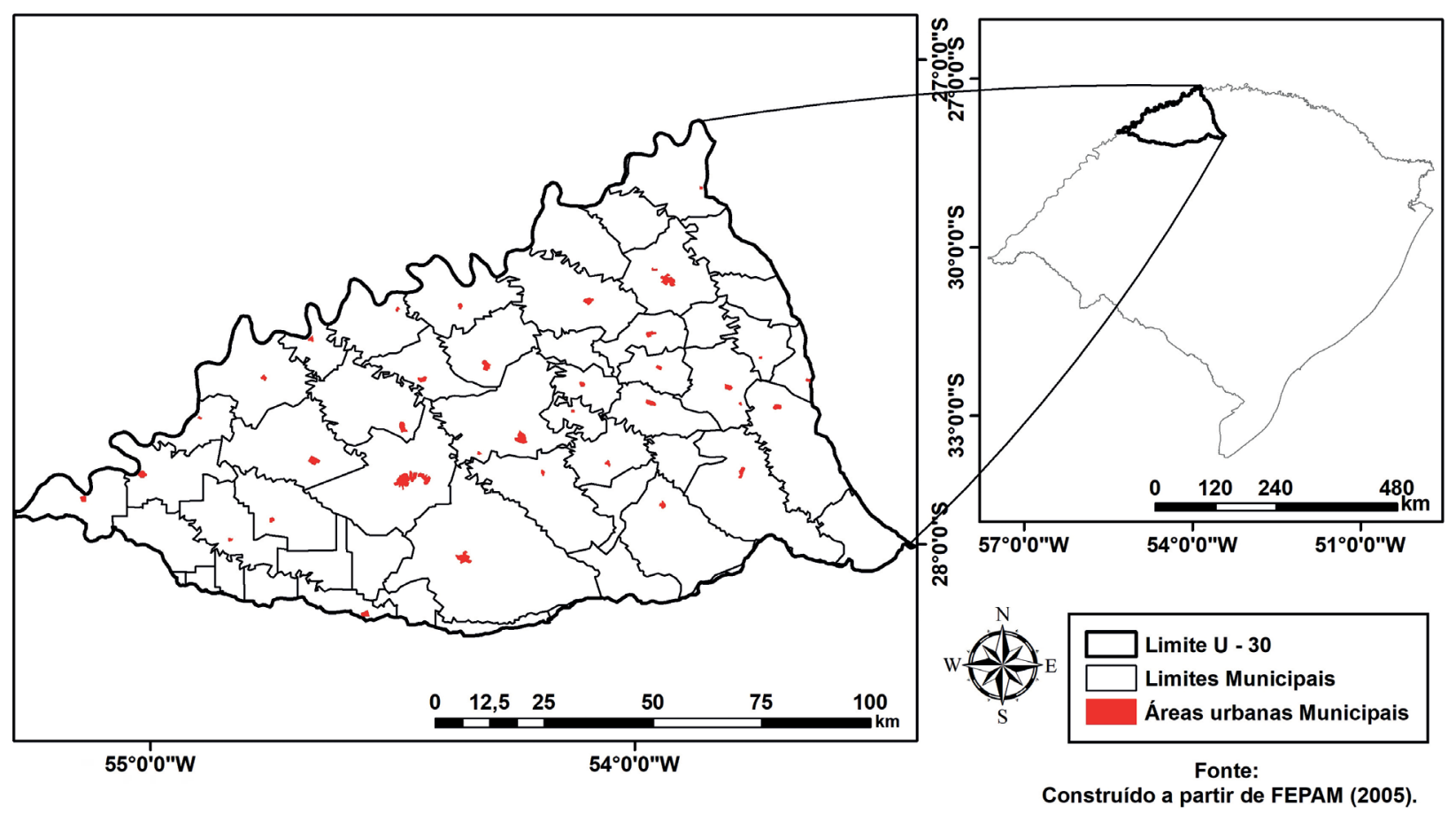

Figura 1. Localização da área de estudo.

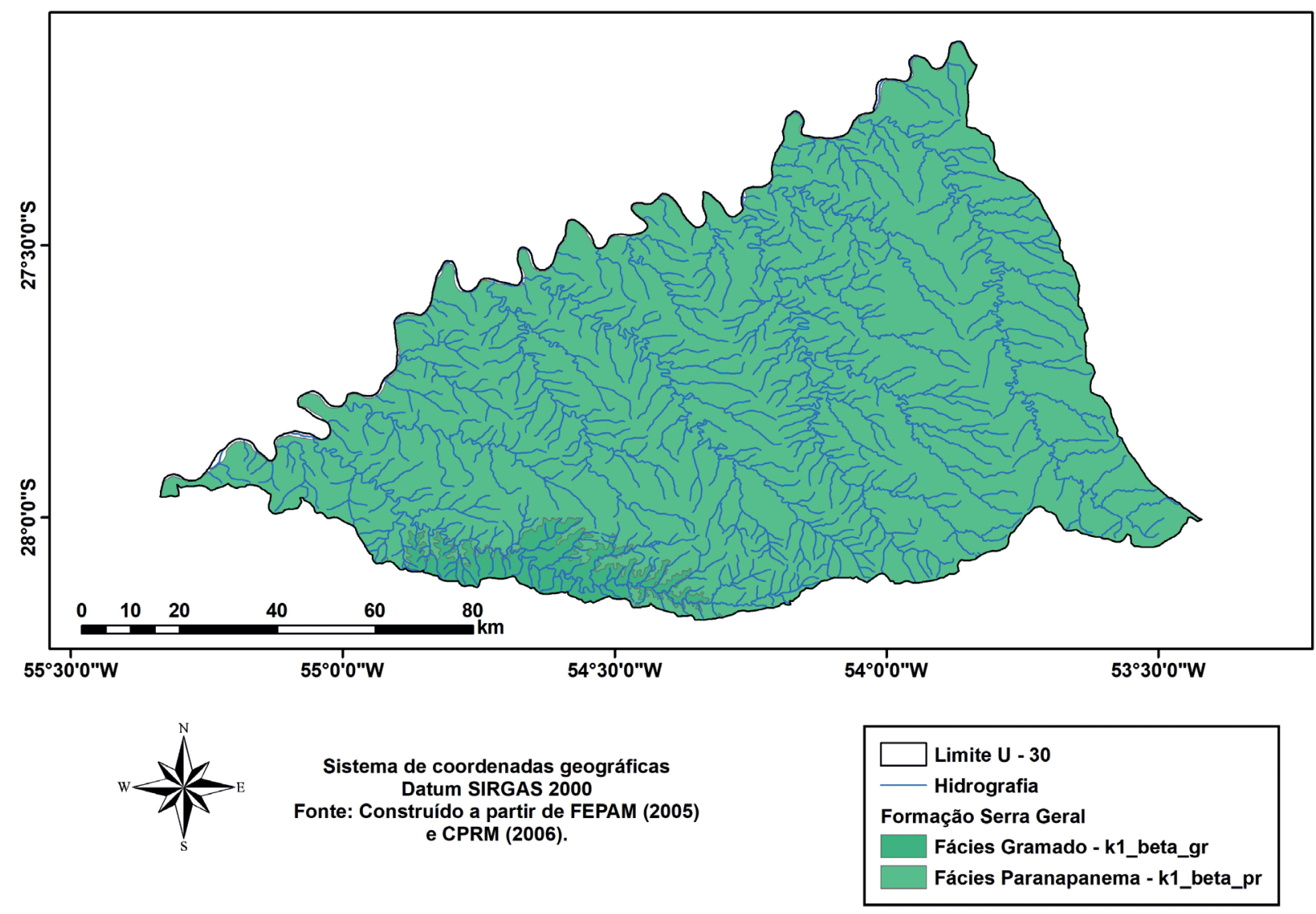

Figura 2. Formações geológicas presentes na área de estudo. 
presença de rochas vulcânicas da Formação Serra Geral Fácies gramado (k1_beta_g), composta das rochas originadas por derrames basálticos maciços, granular homogênea, textura microfanerítica, compacta e de coloração cinza-escuro a cinza-esverdeado (CPRM, 2006).

Em relação à hidrogeologia, conforme Machado e Freitas (2005), existem três tipos de aquífero na área de estudo (Figura 3): o Sistema Aquífero Serra Geral I (sg1), o Sistema Aquífero Serra Geral II (SG2) e, em menores proporções, o Sistema Aquífero Basalto Botucatu (BB). Os autores ressaltam que o sistema $\mathrm{sg} 1$ possui alta a média possibilidade para águas subterrâneas em suas rochas, com porosidade por fraturas constituídas, em sua maioria, de litologias basálticas, amigdaloides e fraturadas, capeadas por espesso solo avermelhado. O sistema SG2, conforme os mesmos autores, possui média a baixa disponibilidade de águas subterrâneas, em razão da existência de rochas com porosidade por fraturas e litologia predominantemente de riolitos, riodacitos e, em menor proporção, basaltos fraturados que dificultam a reserva de águas. Eles ressaltam também que o sistema BB são aquíferos praticamente improdutivos em rochas com porosidade intergranular ou por fraturas.

\section{METODOLOGIA}

\section{Construção do banco de dados}

\section{Informações hidrogeológicas}

Para realizar este estudo, foram utilizadas informações espaciais advindas de diversos bancos de dados governamentais e não governamentais. $\mathrm{O}$ processamento desses dados foi realizado em softwares de Sistema de Informações Geográficas (SIG).

As informações hidrogeológicas (distância do nível freático e material do aquífero) de 953 poços foram obtidas por meio do portal do Sistema de Informações de Águas Subterrâneas (SIAGAS) (CPRM, 2018). Do total de poços pesquisados, 168 estão localizados fora da área objeto deste trabalho, em razão de as informações disponíveis para a

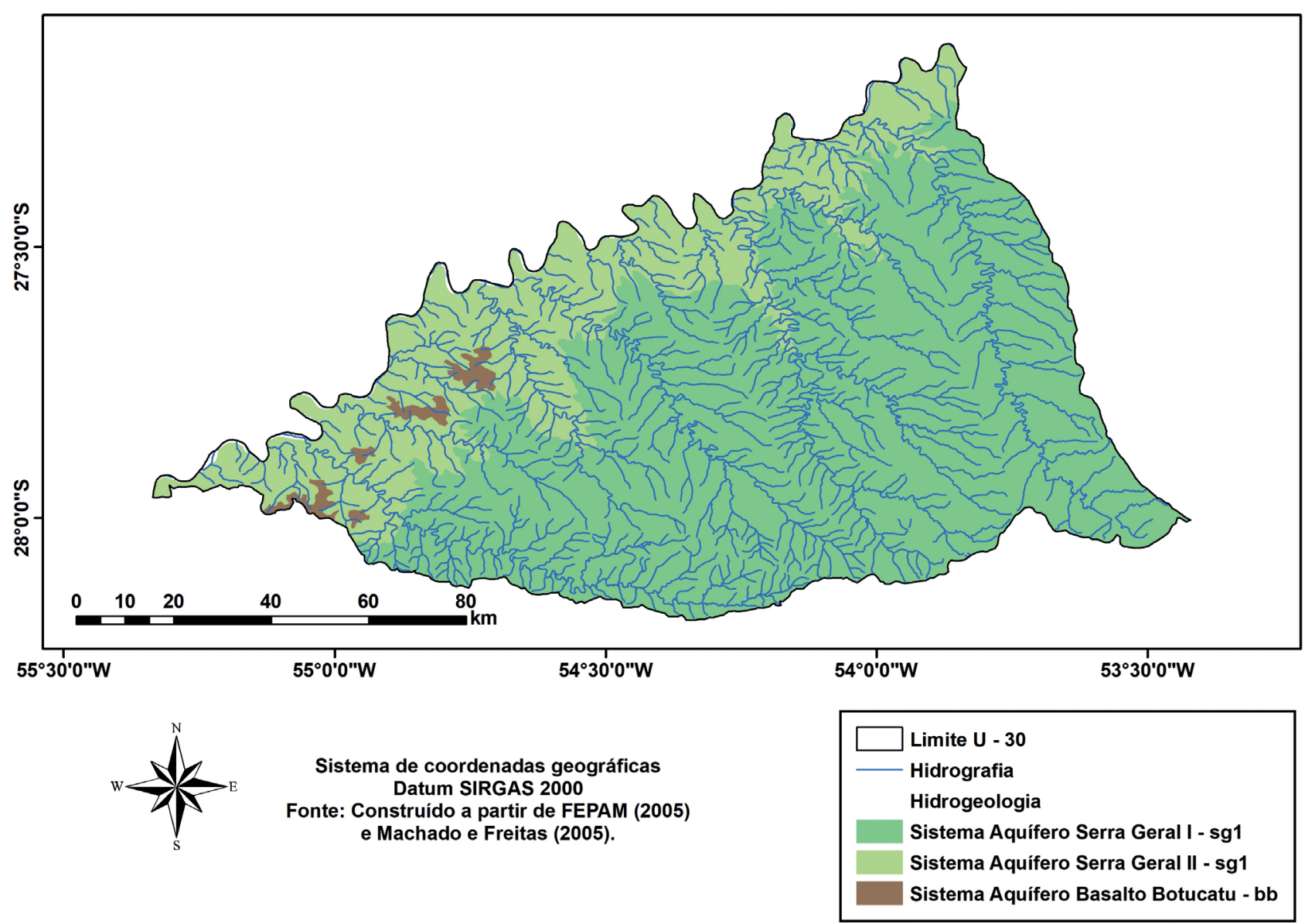

Figura 3. Caracterização hidrogeológica da área de estudo. 
área de estudo não representarem a totalidade das áreas, sendo utilizada a representação regional das informações, conforme proposta por Costa et al. (2011).

Os valores de D (Tabela 1) foram exagerados em dez vezes, conforme sugerido por Veríssimo (2010). Esse autor indica que, com essa extrapolação, ocorre melhor visualização dos parâmetros quando comparados ao parâmetro LU. Para o material do aquífero (Parâmetro A), o basalto foi considerado (CPRM, 2006) predominante em toda a área de estudo, sendo atribuída a nota 60 , conforme proposto por Ribeiro (2005). Em relação à profundidade do nível freático (Parâmetro D), foram utilizadas as classes e as notas dispostas na Tabela 1. Como pode ser observado, quanto menor a profundidade do nível freático, maior será a sua susceptibilidade - em razão da proximidade do nível da água com a superfície - , e consequentemente, maior a sua pontuação.

O parâmetro LU foi obtido com a classificação supervisionada no SIG a partir da identificação, por parte do usuário, de um conjunto de pixels da imagem pertencente a cada uma das classes consideradas no estudo. Em um algoritmo computacional, verifica a semelhança entre a amostra e cada um dos demais pixels da imagem, atribuindo a cada um deles, automaticamente, o valor da classe com que mais se parecem. Nesse caso, foi empregado o algoritmo classificador de máxima verossimilhança, que calcula as distribuições de probabilidade de cada pixel, pertencente a uma classe da amostra, por meio do teorema de Bayes (Richards e Jia, 2006). Nesse processo, foram consideradas as classes e as pontuações apresentadas na Tabela 2. Como pode ser visto, as áreas artificiais receberam a maior pontuação em virtude das atividades desenvolvidas (resíduos e saneamento, por exemplo), quando comparadas às áreas agrícolas e naturais. As imagens utilizadas foram do sensor espectral operation land image (OLI), embarcado no Landsat 8, com resolução espacial de $30 \mathrm{~m}$ (U.S. Department of the Interior, 2013), datada de 6 de julho de 2017 e 26 de julho de 2017, sem a presença de nuvens. As imagens foram obtidas no site Earhexplorer, do United States Geological Survey (USGS).

Tabela 1. Pontuações relativa à profundidade do nível freático.

\begin{tabular}{lc}
\hline Profundidade $(\mathbf{m})$ & Pontuação \\
\hline$<1,5$ & 100 \\
$1,5-4,6$ & 90 \\
$4,6-9,1$ & 70 \\
$9,1-15,1$ & 50 \\
$15,1-22,9$ & 30 \\
$22,9-30,5$ & 20 \\
$>30,5$ & 10 \\
\hline
\end{tabular}

Fonte: adaptado de Ribeiro (2005).
Para realizar a estimativa da recarga subterrânea (Parâmetro R), utilizou-se o valor indicado por Hausman (1995), em que foi considerada recarga média anual de $125 \mathrm{~mm}$. Considerou-se constante o valor de recarga em toda a área de estudo, em virtude da homogeneidade do material rochoso subterrâneo (rochas vulcânicas).

Em relação ao parâmetro topografia (T), foi utilizada as informações disponibilizadas no Shuttle Radar Topography Mission (SRTM) (USGS, 2004), com resolução espacial de $30 \mathrm{~m}$ (USGS, 2004). A pontuação dos valores de topografia está indicada na Tabela 3, em que as menores declividades representam maior susceptibilidade em razão do menor escoamento superficial e, consequentemente, da maior infiltração do líquido pelo solo até a zona vadosa.

As informações pontuais adquiridas in situ foram inseridas no interpolador inverse distance weighted (IDW), ou inverso da distância ponderada, com potência de segunda ordem, para a aquisição das informações de cada parâmetro em toda a bacia hidrográfica. As pontuações dos parâmetros $\mathrm{R}, \mathrm{A}$ e $\mathrm{T}$ foram extrapoladas dez vezes dos valores originais propostos por Ribeiro (2005). Essa extrapolação foi sugerida por Veríssimo (2010), pois representa melhor visualização dos parâmetros quando comparados ao método LU.

\section{Estimativa do índice de susceptibilidade à contaminação do aquífero}

A metodologia de determinação do IS seguiu a proposta de Ribeiro (2005), na qual foi aplicada a Equação 1, na

Tabela 2. Pontuações referentes ao uso e à ocupação do solo.

\begin{tabular}{lc}
\hline Uso e ocupação do solo & Pontuação \\
\hline Áreas agrícolas & \\
$\quad$ Pastagens e áreas agroflorestais, & 50 \\
áreas agrícolas heterogêneas & \\
Áreas artificiais & \\
$\quad$ Áreas urbanas contínuas, aeroportos & 75 \\
Áreas naturais & \\
$\quad$ Ambientes aquáticos & 50 \\
$\quad$ Florestas e zonas seminaturais & 0 \\
\hline
\end{tabular}

Fonte: adaptado de Ribeiro (2005).

Tabela 3. Pontuações referentes à declividade.

\begin{tabular}{lc}
\hline Declividade (\%) & Pontuação \\
\hline$<2$ & 100 \\
$2-6$ & 90 \\
$6-12$ & 50 \\
$12-18$ & 30 \\
$>18$ & 10 \\
\hline
\end{tabular}

Fonte: adaptado de Ribeiro (2005). 
calculadora raster do SIG. Posteriormente, o IS foi classificado conforme a Tabela 4.

$I S=0,186 D+0,212 R+0,256 A+0,121 T+0,222 L U$

Em que:

$\mathrm{D}=$ profundidade do nível freático;

$\mathrm{R}=$ recarga;

$\mathrm{A}=$ material do aquífero;

$\mathrm{T}=$ topografia;

$\mathrm{LU}=$ Land Use (uso do solo).

Tabela 4. Pontuações referentes à vulnerabilidade.

\begin{tabular}{lc}
\hline Valores & Vulnerabilidade \\
\hline$>90$ & Extremamente vulnerável \\
$80-90$ & Muito elevada \\
$70-80$ & Elevada \\
$60-70$ & Moderada a alta \\
$50-60$ & Moderada a baixa \\
$40-50$ & Baixa \\
$30-40$ & Muito baixa \\
$<30$ & Extremamente baixa \\
\hline
\end{tabular}

Fonte: adaptado de Stigter et al. (2006).

\section{RESULTADOS E DISCUSSÃO}

\section{Profundidade do nível freático}

A profundidade do nível freático está apresentada na Figura 4. As zonas claras no mapa apresentam os níveis de profundidade da água subterrânea mais baixos e se referem às pontuações mais altas. Logo, quanto menor a profundidade do aquífero, maior será a sua susceptibilidade à poluição, pois o contato do contaminante com a zona vadosa é facilitado pela baixa distância entre eles.

\section{Recarga e material do aquífero}

Os parâmetros recarga e material do aquífero foram considerados homogêneos em toda a área de estudo. Assim, a Tabela 5 ilustra a classificação desses parâmetros com suas respectivas pontuações.

\section{Topografia}

A declividade presente na área de estudo está apresentada na Figura 5. Segundo Xavier e Coelho Netto (2014), as estruturas

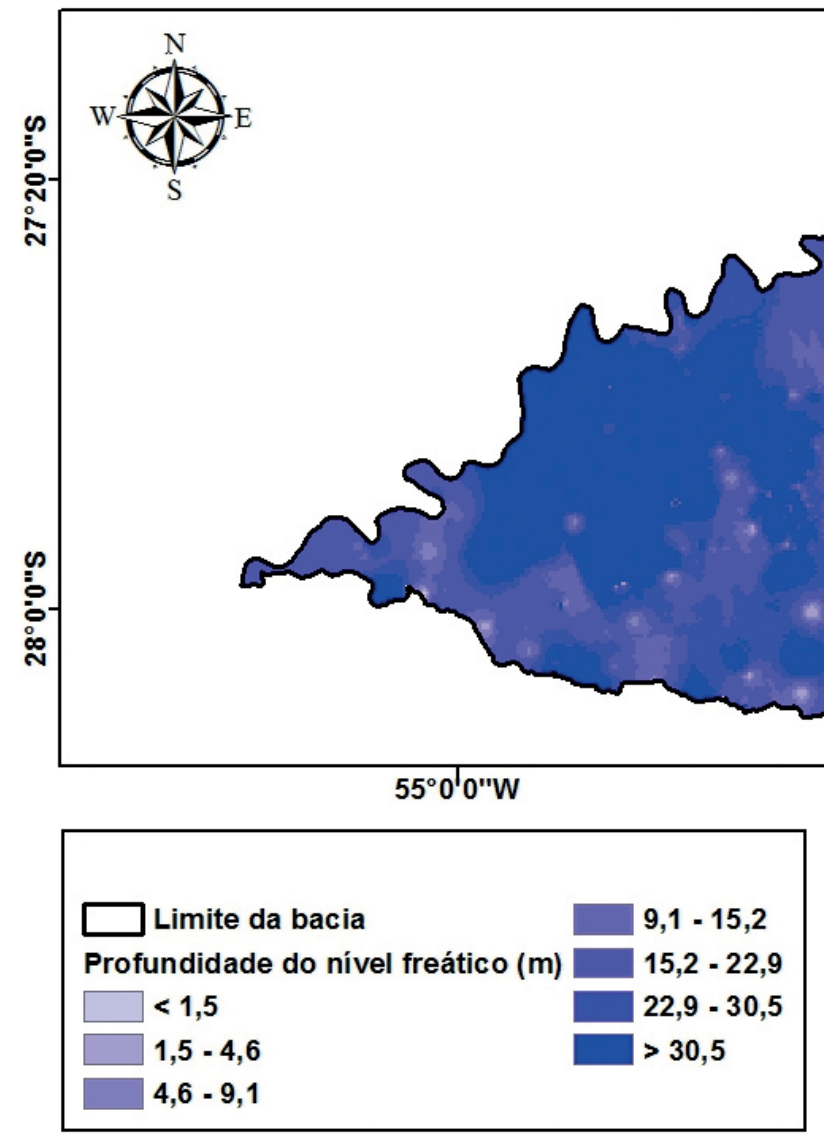

Figura 4. Profundidade do nível freático. 
geológicas influenciam o desenvolvimento do relevo no local, a homogeneidade do substrato geológico e a forte presença de lineamentos estruturais sentido norte-sul, e contribuíram sobremaneira para o recuo da escarpa de falha pela rede de drenagem da bacia. Por isso, ocorre o predomínio de zonas com menor declividade. Essas áreas são mais susceptíveis à poluição em razão do menor escoamento superficial e do maior acúmulo de água, ocasionando maior taxa de infiltração de água e contaminantes, chegando com mais facilidade ao lençol freático.

\section{Uso do solo}

A bacia hidrográfica em questão ocupa uma área de $10.810 \mathrm{~km}^{2}$, sua estrutura agrária é baseada predominantemente nas

Tabela 5. Pontuações referentes à recarga e ao material do aquífero.

\begin{tabular}{lc}
\hline Recarga (mm/ano) & Pontuação \\
\hline $102-178$ & 60 \\
Material do aquífero & Pontuação \\
Basalto & 90 \\
\hline
\end{tabular}

Fonte: adaptado de Ribeiro (2005). pequenas e médias propriedades, com uso do solo destinado principalmente a culturas como soja, milho e, em menor proporção, feijão, aveia e trigo (FEPAM, 2019a). A suinocultura também tem forte influência na região, conforme Brasil (2006). A Figura 6 representa as classes de uso de solo encontradas, ocorrendo a predominância daquelas baseadas em áreas agrícolas, característica comum da região. A Tabela 6 mostra as porcentagens de uso do solo presentes na área de estudo conforme o método IS (Ribeiro, 2005).

Em termos de porcentagem, a área em estudo apresenta cerca de 78,20\% de pastagens e áreas agroflorestais, 20,07\% de florestas e zonas seminaturais, $0,92 \%$ de área urbana e $0,81 \%$ de zonas naturais. A elevada porcentagem da classe de pastagens e áreas agroflorestais ocorre em razão das atividades agrícolas e agropecuárias desenvolvidas na região, visto que no mês de obtenção das imagens (julho) a cobertura de solo nessa região geralmente é feita com plantio de trigo, nabo, azevém e aveia.

\section{Estimativa do índice de susceptibilidade}

A estimativa do IS (Ribeiro, 2005) está ilustrada na Figura 7. É importante ressaltar as atividades econômicas

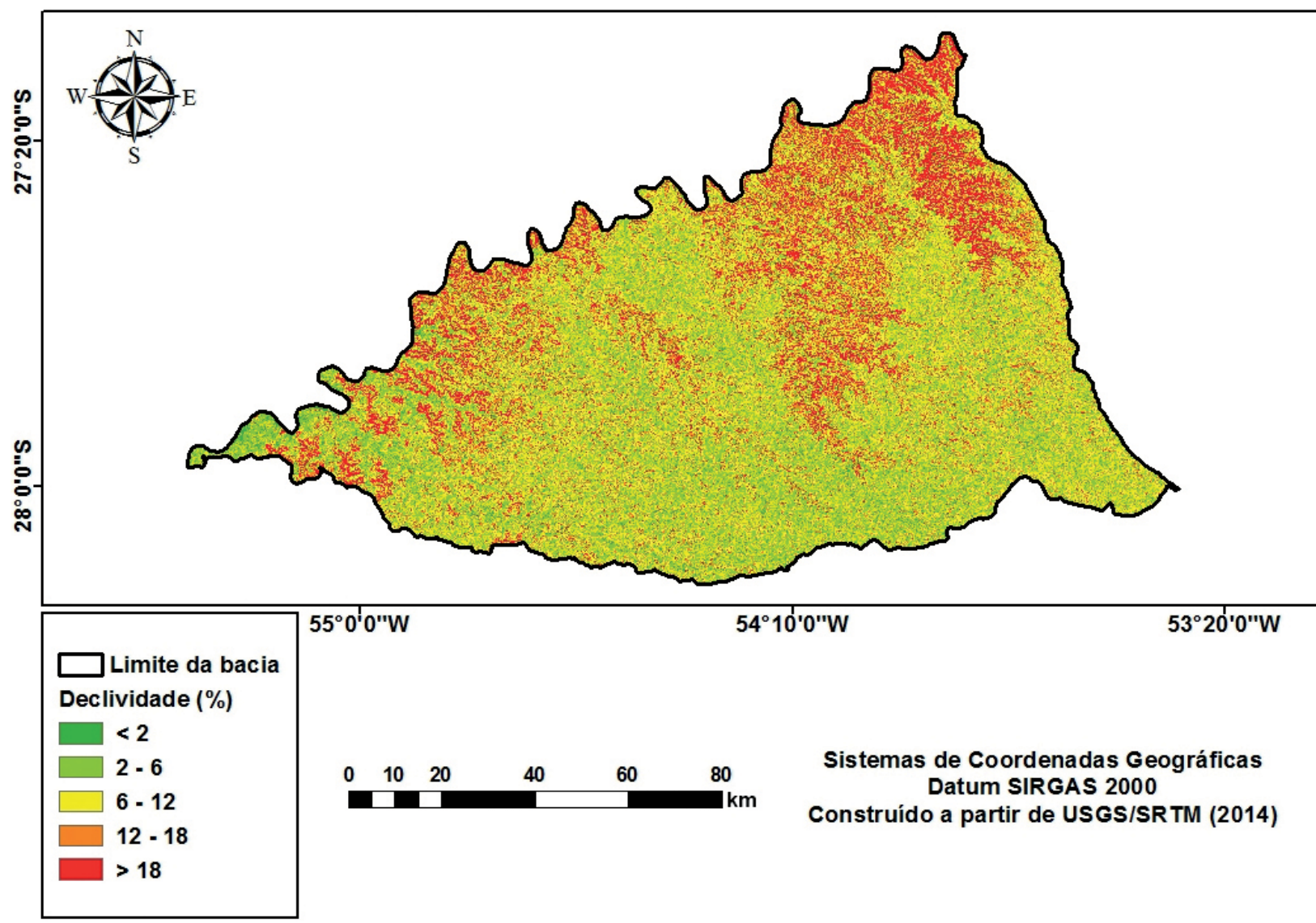

Figura 5. Declividade presente na área de estudo. 
desenvolvidas, em que se destaca a suinocultura, aliada à agricultura e à pecuária. Segundo Poeta et al. (2014), a mesorregião noroeste do Estado do Rio Grande do Sul na qual está inserida a bacia em estudo - representa 56\% das granjas comerciais e $34,10 \%$ das granjas integradas de suínos do estado.

Pereira et al. (2009) afirmam que a suinocultura tem alto potencial poluidor em virtude dos efluentes, que causam contaminação e degradação do ar, dos recursos hídricos e do solo. Eles dizem também que o lançamento indiscriminado de dejetos não tratados em contato com o solo e a água pode trazer desconforto ou doenças à população, assim como seu lançamento em corpos hídricos pode causar mortandade da vida aquática e eutrofização. Aliado a isso, destaca-se a relevância do tema, visto do alto potencial contaminante da atividade suinícola na região, visto que, segundo a Fundação Estadual de Proteção Ambiental Luiz Carlos Roessler (FEPAM, 2019b), na área de estudo existem 1.498 atividades desse tipo cadastradas.
Neste estudo, as classes de IS (Ribeiro, 2005) foram categorizadas entre muito baixa $(4,895 \%$ da área) e muito elevada $(0,004 \%$ da área). Estudos realizados por Borba et al. (2016) e Lunardi (2018) no SASG (mesma hidrogeologia encontrada na área em estudo) apontam classes de susceptibilidade entre muito baixa e elevada. Tal constatação indica que as condições homogêneas da região, principalmente o uso do solo (predominância de pastagens e áreas agrícolas, característica comum da região), além da recarga e do material do aquífero (parâmetros com maior peso na

Tabela 6. Porcentagem de uso do solo obtida para a área de estudo.

\begin{tabular}{lcc}
\hline Classe de uso & Área (Ha) & \% da área \\
\hline Zonas naturais & $8.804,64$ & 0,81 \\
Pastagens e áreas agroflorestais & $846.645,98$ & 78,20 \\
Florestas e zonas seminaturais & $217.308,81$ & 20,07 \\
Áreas urbanas contínuas & $9.949,77$ & 0,92 \\
\hline
\end{tabular}

Fonte: adaptado de Ribeiro (2005).

Figura 6. Mapa das classes de uso do solo e total de suinocultura por município. 
equação do IS), fornecem susceptibilidade com características comuns entre as áreas.

Em outras regiões, estudos desenvolvidos por Ribeiro (2005) e Peralta et al. (2005) no Sistema Aquífero dos Gabros de Beja, em Portugal, encontraram IS predominante entre médio e alto. No Equador, Ribeiro et al. (2017) encontraram IS variando de extremamente baixo a elevado. A estimativa da susceptibilidade para o presente estudo está ilustrada na Figura 7, enquanto a Tabela 7 indica as classes de IS e suas respectivas áreas.

Os valores mais altos do IS estão próximos às áreas urbanas, o que pode estar relacionado ao uso e à ocupação do solo, sendo um dos parâmetros que mais influenciam no método (maior ponderamento). Isso ocorre em razão do potencial contaminante que essa classe possui, por meio da presença de sistemas de coleta de esgotos, cemitérios, postos e combustíveis e locais de disposição de resíduos sólidos, por exemplo. Segundo Foster et al. (2002; 2006), os cemitérios apresentam reduzido potencial de geração de carga contaminante no subsolo, e os postos de combustíveis e as redes de esgoto, moderado potencial. Assim, destaca-se o potencial risco que essas atividades representam no meio subterrâneo.

Tabela 7. Classes de índice de susceptibilidade e suas respectivas áreas obtidas para a área de estudo.

\begin{tabular}{lcc}
\hline Classe de susceptibilidade & Área (Ha) & \% da área \\
\hline Muito baixa & $52.465,68$ & 4,89 \\
Baixa & $179.039,88$ & 16,70 \\
Moderada a baixa & $600.284,88$ & 56,01 \\
Moderada a alta & $234.478,17$ & 21,87 \\
Elevada & $5.431,50$ & 0,50 \\
Muito elevada & 47,52 & 0,004 \\
Total & $1.071 .747,63$ & 100
\end{tabular}

Fonte: adaptado de Stigter et al. (2006).

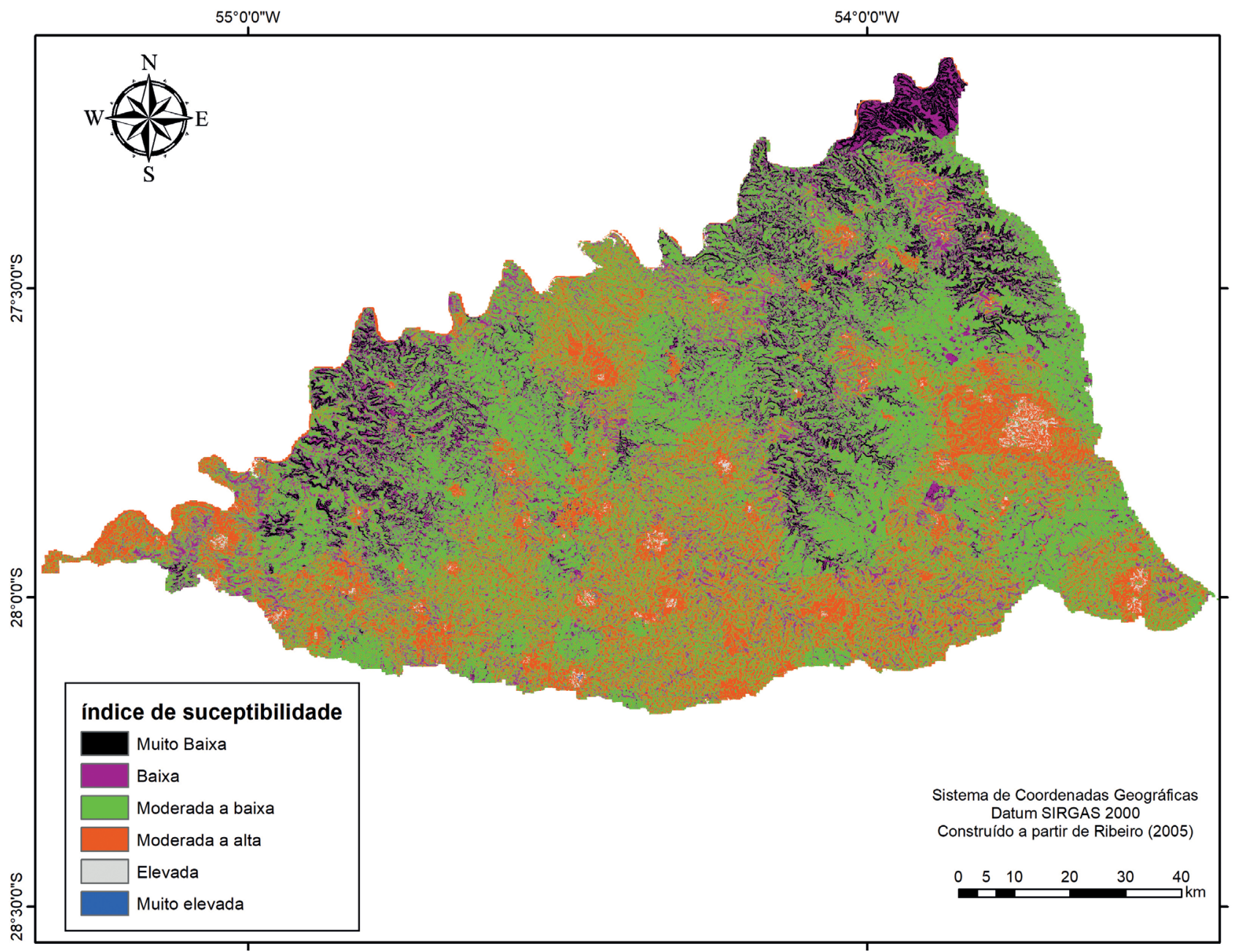

Figura 7. Estimativa índice de susceptibilidade para a área de estudo. 


\section{CONCLUSÃO}

A aplicação do método IS para estimativa da susceptibilidade à contaminação da Bacia hidrográfica dos rios Turvo, Santa Rosa e Santo Cristo indicou que a susceptibilidade variou de muito baixa ( $4,90 \%$ da área) a muito elevada $(0,004 \%)$, com predomínio da classe moderada a baixa $(56,01 \%)$. Isso indica que a área apresenta condições naturais de proteção, em sua maior porção. Ressalta-se a relevância de um estudo mais elaborado, levando em consideração a locação das atividades com potencial contaminador e o cruzamento com os resultados deste trabalho, evidenciando quais áreas são mais indicadas para o desenvolvimento das atividades.

As áreas mais suscetíveis à contaminação estão localizadas próximas às áreas urbanas, o que demonstra a importância de uma rede de esgotos efetiva, bem como o adequado tratamento dos resíduos gerados pelas populações ocupantes da bacia hidrográfica dos rios em questão, além do efetivo manejo agrícola e o correto descarte ou aproveitamento dos resíduos gerados pela suinocultura. Esses resultados indicam o potencial da metodologia adotada no suporte para tomada de decisão relacionada ao meio ambiental, como a criação de planos diretores municipais ou planos de bacia hidrográfica.

\section{REFERÊNCIAS}

Agência Nacional de Águas (ANA). (2015a). Atlas água e esgotos. Brasília, ANA.

Agência Nacional de Águas (ANA). (2015b). Panorama das águas: águas subterrâneas. Disponível em: $<\mathrm{http}: / / \mathrm{www} 3$. ana.gov.br/portal/ANA/panorama-das-aguas/quantidade-daagua/agua-subterranea $>$. Acesso em: 11 fev. 2019.

Aller, L., Bennet, T., Lehr, J. H., Petty, R. J. (1987). DRASTIC: a standardized system for evaluating groundwater pollution potential using hydrogeologic settings. 600/2-87/035. EPA., 58p.

Anane, M., Abidi, B., Lachaal, F., Limam, A., Jellali, S. (2013). GIS-based DRASTIC, Pesticide DRASTIC and the Susceptibility Index (SI): comparative study for evaluation of pollution potential in the Nabeul-Hammamet shallow aquifer, Tunisia. Hydrogeology Journal, 21(3), 715-731. https://doi.org/10.1007/s10040-013-0952-9

Borba, W. F., Silva, J. L. S., Allasia, D. G., Rosa, C. R., Favaretto, J. R., Ribeiro, L. F. T. (2016). Geoprocessamento Aplicado à Determinação do Índice de Susceptibilidade das Captações por Poços Tubulares do Sistema Aquífero Serra Geral em Frederico Westphalen - Rio Grande do Sul - Brasil. Anuário do Instituto de Geociências, 39(3), 79-88. https:// doi.org/10.11137/2016_3_79_88
Brasil. Ministério do Meio Ambiente. (2006). Caderno da região hidrográfica do Uruguai. Brasília, MMA. 128 p.

Chrispim, Z. M. P. (2016). Análise da Vulnerabilidade e Caracterização Hidrogeoquímica dos Aquiferos Livres Rasos da Parte Emersa da Bacia Sedimentar de Campos. Tese (Doutorado). Campos dos Goytacazes: Programa de Pós-Graduação em Engenharia Civil, Universidade Estadual do Norte Fluminense Darcy Ribeiro, 24 p.

Companhia de Pesquisa de Recursos Minerais (CPRM). (2004). Mapa de Domínios e Subdomínios Hidrogeológicos do Brasil - Escala 1:2.500.000. Porto Alegre, CPRM.

Companhia de Pesquisa de Recursos Minerais (CPRM). (2006). Mapa Geológico do Rio Grande do Sul, Escala 1:750.000. Brasília, CPRM.

Companhia de Pesquisa de Recursos Minerais (CPRM). (2018). Sistema de Informações de Águas Subterrâneas (SIAGAS). Disponível em: <http://siagasweb.cprm.gov. br/layout/>. Acesso em: 20 maio 2015.

Costa, M. L. M. C., Ribeiro, M. M. R., Rêgo, J. C. R., Albuquerque, J. do P. T. (2011). Preposição de critérios de outorga para águas subterrâneas - Brasil. Revista Brasileira de Recursos Hídricos, 16(1), 105-113. https://doi.org/10.21168/ rbrh.v16n1.p105-113

Francés, A., Paralta, E., Fernandes, J., Ribeiro, L. (2001). Development and application in the Alentejo region of a method to assess the vulnerability of groundwater to diffuse agricultural pollution: the susceptibility index. $3^{\text {rd }}$ International Conference on Future Groundwater Resources at Risk (FRG'01). Proceedings... Lisboa, CVRM/IST, p. 35-44.

Foster, S., Hirata, R., Gomes, D., D’Elia, M., Paris, M. (2002). Groundwater quality protection: a guide for water utilities, municipal authorities, and environment agencies. Washington: The World Bank, $114 \mathrm{p}$.

Foster, S., Hirata, R., Gomes, D., D’Elia, M., Paris, M. (2006). Proteção da qualidade da água subterrânea: um guia para empresas de abastecimento de água, órgãos municipais e agências ambientais. Washington: Banco Mundial, 115 p.

Freitas, M. A., Binotto, R. B., Nanni, A. S., Rodrigues, A. L. M., Bortoli, C. R. (2012). Avaliação do Potencial Hidrogeológico, Vulnerabilidade Intrínseca e Hidroquímica do Sistema Aquífero Serra Geral no Noroeste do Estado do Rio Grande do Sul - Brasil. Revista Brasileira de Recursos Hidricos, 17(2), 31-41. https://doi.org/10.21168/ rbrh.v17n2.p31-41 
Fundação Estadual de Proteção Ambiental Luiz Carlos Roessler (FEPAM). (2005). Arquivo digital para uso em SIG - Base cartográfica digital do RS 1:250. Disponível em: $<$ http://www.fepam.rs.gov.br/biblioteca/geo/bases_geo. asp>. Acesso em: 10 fev. 2019.

Fundação Estadual de Proteção Ambiental Luiz Carlos Roessler (FEPAM). (2019a). U30 - Turvo/Santa Rosa/Santo Cristo. FEPAM. Disponível em: $<$ http://www.fepam.rs.gov. br/qualidade/bacia_uru_turvo.asp $>$. Acesso em: 29 out. 2019.

Fundação Estadual de Proteção Ambiental Luiz Carlos Roessler (FEPAM). (2019b). Licenciamento Ambiental. FEPAM. Disponível em: <http://www.fepam.rs.gov.br/ licenciamento/Area1/default.asp>. Acesso em: 29 out. 2019.

Governo do Estado do Rio Grande do Sul. (2011). Elaboração de serviço de consultoria relativo ao processo de planejamento no uso da água na bacia hidrográfica dos rios Turvo - Santa Rosa - Santo Cristo - Enquadramento. Relatório Técnico 1: Atividades preliminares - RT1. Porto Alegre, Engeplus, 391 p.

Guiguer, N., Kohnke, M. W. (2001). Métodos para determinação da vulnerabilidade de aquíferos. XII Congresso Brasileiro de Águas Subterrâneas. 13 p.

Hausman, A. (1995). Esboço Hidrogeológico do Rio Grande do Sul - Brasil. Acta Geologica Leopoldensia, 37-71.

Heath, R. C. (1982). Basic ground-water hydrology. Water supply paper. Denver: USGS, 86 p.

Lunardi, S. (2018). Susceptibilidade a contaminação do Aquífero Serra Geral no município de Sarandi-RS. TCC (Graduação). Frederico Westphalen: Graduação em Engenharia Ambiental e Sanitária, Universidade Federal de Santa Maria.

Machado, J. L. F., Freitas, M. A. (2005). Projeto mapa hidrogeológico do Estado do Rio Grande do Sul: escala 1:750.000, relatório final. Porto Alegre, CPRM, 65 p.

Motter, A. F.C., Foleto, E. M. (2010). Um olhar sobre a gestão dos recursos hídricos: o caso do comitê de gerenciamento da Bacia Hidrográfica dos Rios Santa Rosa, Santo Cristo e Turvo - noroeste do Rio Grande do Sul. Perspectiva, 34(126), 143-155.

Nanni, A. S., Freitas, M. A., Tedesco, M. A., Binotto, R. B. (2005). Vulnerabilidade natural e risco de contaminação do aquífero Serra Geral pela suinocultura na região das missões - RS. Brasil. XVI Simpósio Brasileiro de Recursos Hídricos. Anais... João Pessoa. p. 70-85.
Peralta, E., Francés, A., Ribeiro, L. F. (2005). Avaliação da vulnerabilidade do Sistema Aquífero dos Gabros de Beja e análise crítica das redes de monitorização no contexto da directiva quadro da água. VII Simpósio de Hidráulica e Recursos Hídricos dos Países de Língua Oficial Portuguesa. Anais... Évora, APRH, p. 14.

Peralta, E., Oliveira, M., Batista, S., Francés, A., Ribeiro, L., Cerejeira, M. J. (2001). Aplicação de SIG na avaliação da vulnerabilidade aquífera e cartografia da contaminação agrícola por pesticidas e nitratos na região do Ribatejo. Seminário a Hidroinformática em Portugal. Anais... Lisboa, LNEC, p. 16.

Pereira, E. R., Demarchi, J. J. A. A., Budiño, F. E. L. (2009). A questão ambiental e os impactos causados pelos efluentes da suinocultura - Brasil. Infobibos, Informações Tecnológicas, 7(1), 18-20. Disponível em: <http://www. infobibos.com/Artigos/2009_3/QAmbiental/index.htm>. Acesso em: 3 fev. 2020.

Poeta, A. S., Santiago Neto, W., Vergara, E. N., Cavagni, G. M., Correa, A. M. R., Santos, D. V. dos. (2014). Panorama da suinocultura no Rio Grande do Sul - Informativo técnico número 7, ano 5. Porto Alegre, SEAPA.

Rebouças, A. C. (2008). Importância da água subterrânea. In: F. A. C. Feitosa, J. Manoel Filho, E. C. Feitosa, J. G. A. Demetrio. Hidrogeologia: conceitos e aplicações. Rio de Janeiro: CPRM/LABHIDRO, 812 p.

Rebouças, A. C., Braga, B., Tundisi, J. G. (2002). Águas doces do Brasil: capital ecológico, uso e conservação. São Paulo: Escrituras, 732 p.

Ribeiro, L. F. (2005). Um novo índice de vulnerabilidade específico de aquíferos - formulações e aplicações. VII Simpósio de Hidráulica e Recursos Hídricos dos Países de Lingua Oficial Portuguesa. Anais... Évora, APRH, p. 14.

Ribeiro, L., Pindo, J. C., Dominguez-Granda, L. (2017). Assessment of groundwater vulnerability in the Daule aquifer, Ecuador, using the susceptibility index method. Science of the Total Environment, 574, 1674-1683. https:// doi.org/10.1016/j.scitotenv.2016.09.004

Richards, J. A., Jia X. (2006). Remote Sensing Digital Image Analysis: An Introduction. Berlim: Springer.

Secretaria do Meio Ambiente do Estado do Rio Grande do Sul (SEMA) (2004). Mapa das Bacias Hidrográficas e Municipios do Rio Grande do Sul. Porto Alegre, SEMA. 
Stigter, T. Y., Ribeiro, L., Carvalho Dill, A. M. M. (2006). Evaluation of an intrinsic and a specific vulnerability assessment method in comparison with groundwater salinisation and nitrate contamination levels in two agricultural regions in the south of Portugal. Hydrogeology Journal, 14, 79-99. https://doi.org/10.1007/s10040-004-0396-3

United States Geological Survey (USGS). (2004). Shuttle Radar Topography Mission, 1 Arc Second Scene Srtm1s28w054v3, Unfilled Unfinished 2.0, Global Land Cover Facility. Maryland: University of Maryland.

U.S. Department of the Interior. NASA Landsat Program. (2013). Landsat ETM+ cena LC82230792013112LGN01,
L1T, USGS, Sioux Falls. Disponível em: < http://earthexplorer. usgs.gov/>. Acesso em: 10 dez. 2018.

Veríssimo, A. C. F. (2010). Hidroquímica, vulnerabilidade e protecção do aquífero de torres vedras. Dissertação (Mestrado). Lisboa: Programa de Pós-Graduação em Engenharia Geológica, Universidade de Lisboa, 140 p.

Xavier, R. A., Coelho Netto, A. L. (2014). Caracterização Geomorfológica da bacia do Rio Turvo-RJ: Médio Vale do Rio Paraíba do Sul (MVPRS)/Geomorphological characterization of the Turvo river basin-RJ: The Middle Paraíba do Sul River Valley (MVRPS). Revista Brasileira de Geomorfologia, 15(1), 35-45. https://doi.org/10.20502/rbg.v15i1.372 